\title{
Uncovering intermittent entropion
}

This article was published in the following Dove Press journal:

Clinical Ophthalmology

19 February 2013

Number of times this article has been viewed

\author{
Sidney Julio Faria-e-Sousa' \\ Monike de Paula Gomes \\ Vieira' \\ Jailton Vieira Silva ${ }^{2}$ \\ 'University of São Paulo, São Paulo,

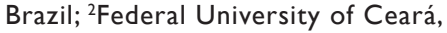 \\ Ceará, Brazil
}

Purpose: Intermittent entropion may go unnoticed by both patient and physician, in some instances resulting in ocular surface disorders. Unfortunately, the current work-up used to diagnose entropion is not always sufficient to uncover the problem. We present an alternative diagnostic test.

Cases: Two case reports are presented showing different situations where the current methods for detection of entropion failed and the diagnosis was only made with an alternative diagnostic test.

Results: The common denominator of both cases is an intermittent entropion which could not be detected by the conventional clinical work-up. Diagnosis was easy however using the "TIE test" described in the text.

Conclusion: The important clues for suspicion of intermittent entropion are an intermittent foreign-body sensation in the eye accompanied with central punctate keratopathy. The TIE test is a simple and useful test for the diagnosis of intermittent entropion.

Keywords: eyelids, lid abnormality, TIE test

\section{Introduction}

Acute spastic entropion usually occurs after any ocular surgery or an inflammatory condition associated with eyelid edema and/or orbicular spasm. In both cases, a strong contraction of the orbicularis muscle makes its preseptal portion overlap the pretarsal portion, causing the eyelid to roll inward toward the eye. ${ }^{1}$ If the entropion is intermittent, it may go unnoticed by both patient and physician. When recognized, the use of adhesive tape to maintain the correct position of the eyelids or botulinum toxin to relieve the spasm of the orbicularis may cure the problem. ${ }^{2}$ Permanent entropion is usually associated with dehiscence or attenuation of capsulopalpebral fascia and/or eyelid laxity. In terms of diagnosis, the main complaint of intermittent entropion is an intermittent foreign-body sensation in the eye, with or without the perception of the eyelid margin inversion. A mild ptosis of the upper eyelid and a small preseptal swelling of the lower eyelid are usually present. Obviously, the main sign of the abnormality is the inward rolling of the eyelid toward the eye. Thus, if intermittent entropion is suspected, the patient should be asked to tightly close the eyelids. The goal is to duplicate the pathophysiology of the anomaly. Indirect signs of a predisposition to entropion can also help in the diagnosis. ${ }^{3-5}$ These include: (1) observation of the excursion of the lower eyelid in the downward gaze - impaired movement indicates a weakening or disinsertion of the eyelid retractors; (2) examination of the inferior fornix - dehiscent retractors appear as a delicate white line between the tarsus and 
the fornix; (3) horizontal eyelid laxity test, in which the eyelid is pulled forward - in involutional entropion, the amount of the eyelid dislocation away from the globe can be up to $15 \mathrm{~mm}$, the normal being 2-3 mm; and (4) snap-back test, in which the eyelid is pushed downward and released it should readily return to its original position without the aid of a blink. In involutional entropion, the eyelid hangs down below its normal position until a blink returns it to the original position. In terms of slit-lamp biomicroscopy, the main finding is a central corneal punctate epitheliopathy reflecting the friction of the inverted eyelid to the cornea. However, when none of these tests uncover the intermittent entropion, what more can be done? The objective of this paper is to show two cases of intermittent entropion, which could not be detected by the conventional clinical work-up, that were easily diagnosed with an alternative diagnostic test for similar cases.

\section{Case reports Case I}

An 85-year-old woman had been submitted to corneal transplantation in the left eye twice in 2 years. She complained of pain and decreased vision in this eye, the only eye with useful vision. She was recovering from a viral conjunctivitis that started 15 days prior. Corrected visual acuity was: OD (right eye) = light perception and OS (left eye) $=20 / 200$. Slit-lamp biomicroscopy revealed an extensive central corneal de-epithelialization, without signs of infection. It started with an intermittent foreign body sensation in the eye that became worse with time. Her granddaughter informed us that the lower eyelid of the affected eye seemed to turn inwards occasionally. Backed by specific reasons for suspecting an intermittent entropion, triggered by conjunctival inflammation, several unsuccessful attempts were made to induce the phenomenon by asking the patient to tightly close the eyelids. Studying the indirect signs for disclosing predisposition to entropion (eg, excursion of the lower eyelid, dehiscence of the eyelid retractors, eyelid laxity) was not helpful. The diagnosis was eventually made with the test presented below. To protect the corneal epithelium, a therapeutic contact lens was prescribed. One month later, the contact lens was removed, since the cornea was fully epithelialized; the entropion could no longer be elicited by the test that diagnosed it. Corrected visual acuity was: $\mathrm{OS}=20 / 40$. Eight months later, the patient returned with a persistent entropion, infectious corneal ulcer, and graft rejection. She underwent surgery for entropion and a new corneal transplantation. The eyelid abnormality was successfully corrected, but the graft failed, resulting in a corrected visual acuity of 20/400.

\section{Case 2}

A 37-year-old auto-mechanic male complained that in the last 6 months whenever leaning over the engine of a car he would start to feel a foreign body sensation in the left eye. The sensation could be halted by rubbing the eye. The problem was worsening with time with ever more frequent episodes. He also had the impression that the eye was becoming progressively smaller. Corrected visual acuity was: $\mathrm{OD}=20 / 20$ and $\mathrm{OS}=20 / 25$. Upon inspection, the affected eye had a mild ptosis of the upper eyelid and a moderate preseptal swelling of the lower eyelid. Slit-lamp biomicroscopy of the central cornea revealed a narrow strip of punctate staining with fluorescein, suggestive of abrasion. The conjunctiva presented a mild to moderate hyperemia. Suspecting intermittent entropion, we made several unsuccessful attempts to induce the phenomenon, asking the patient to tightly close the eyelids. Studying the indirect signs for disclosing predisposition to entropion was not helpful. The diagnosis was eventually made with the test presented below. The patient was instructed to use an adhesive tape in the lower eyelid to prevent its inward turning. On the next visit, 15 days later, the patient reported significant improvement of symptoms. We referred him to an oculoplastic surgeon for a second opinion. Three months later, the patient returned informing us that with the use of the tape for about 40 days his eye had healed completely. Therefore, he did not seek additional medical help. The test that diagnosed the entropion was now negative. The punctate staining disappeared. Corrected visual acuity was 20/20 in both eyes. We have since lost contact with the patient.

The common denominator of the above cases is an intermittent entropion which could not be detected by the conventional clinical work-up. However, they could be easily diagnosed with the test described below.

\section{Test of induced entropion (TIE test)}

The objective of the TIE test is to force the preseptal portion of the orbicularis muscle to override its pretarsal portion and in so doing induce the inward turning of the eyelid. It comprises the following five steps.

1. With the eye anesthetized topically, ask the patient to look down (Figure 1A).

2. Pinch the lower eyelid with the index finger and thumb (Figure 1B). 


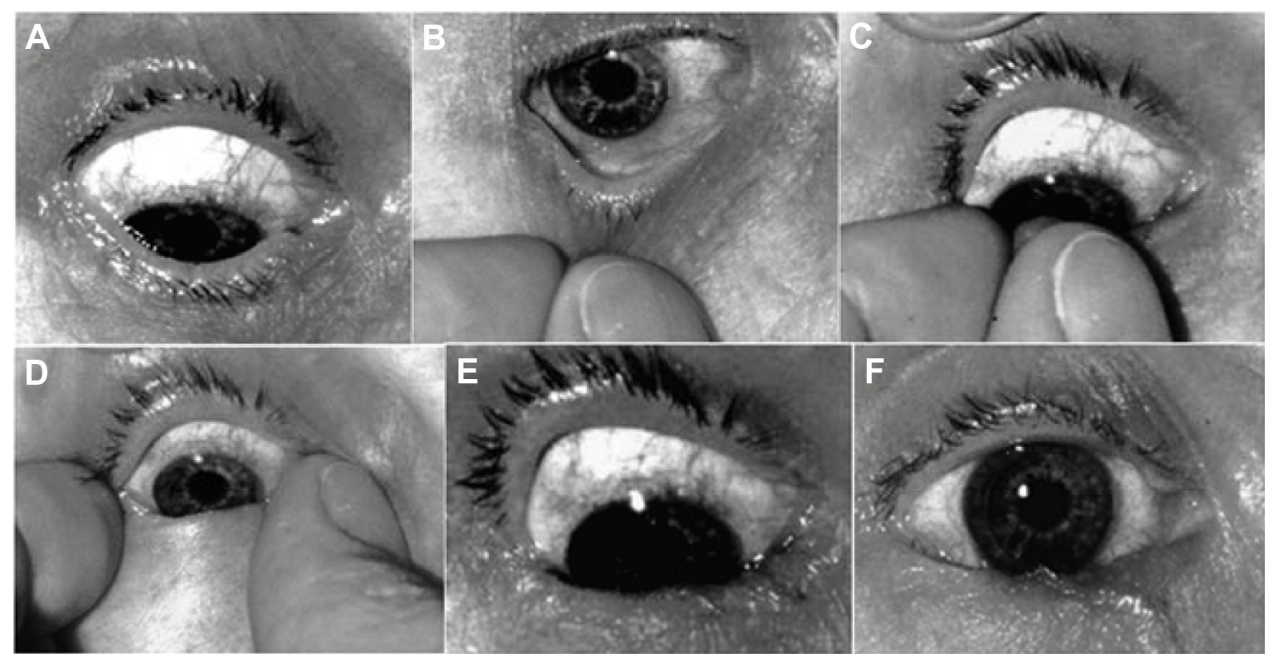

Figure I Steps of the TIE test. (A) Eye looking down. (B) Pinching of the lower eyelid. (C) Skin being rolled over the tarsal edge. (D) Spreading the eyelid against the eyeball. (E) The result after releasing the eyelid. (F) Stable entropion.

3. Lift the skin and roll it over the tarsal edge, pressing it against the eyeball (Figure 1C).

4. Spread the eyelid against the eyeball by straightening out the finger and thumb (Figure 1D).

5. Release the eyelid, and observe the result (Figure 1E).

6. If an entropion is induced, make sure it is stable (Figure 1F).

The test is easily performed by a single examiner as long as the eye is well anesthetized and the patient understands that he or she has to keep the eyes looking fully downwards during the test. Sometimes it is easier to ask the patient to raise the chin high, keeping the line of sight straight ahead.

\section{Discussion}

Intermittent entropion seems to be a rare event in a corneal service. We diagnosed only ten cases in 20 years. Two of them, including Case 2, reverted spontaneously. Seven cases were successfully corrected with surgery of the lower eyelid in different oculoplastic services. One case (Case 1) regressed for a while and then returned, resulting in severe loss of vision. The cases where the condition reverted spontaneously included patients under 40 years of age with no evident signs of a predisposition to entropion. However, both had a mild preseptal swelling of the lower eyelid. We do not know whether these cases would have evolved to a constant entropion if not treated properly. Case 1 was chosen to show the potential risks of an intermittent entropion. Case 2 was chosen because of the peculiarity of the patient's age, complaint, and progression. Important clues for suspicion of intermittent entropion and therefore for the use of this test are an intermittent foreign-body sensation in the eye accompanied with central punctate keratopathy. Different conditions can cause this clinical combination: blepharitis, trichiasis, foreign body in the superior conjunctival sac, and small lesions of Mulluscum contagiosum hidden under the eyelashes. All of these must be ruled out before suspecting intermittent entropion. Preseptal swelling of the lower eyelid may be another clue, since it was present in all of our cases. The test of induced entropion was taught to one of the authors about 20 years ago by Fred M Wilson of the Indiana University. Since then, it has been successfully used in our corneal service under the name of "TIE test of Wilson." This name intentionally emphasizes that its primary goal is the induction of an entropion. However, there is the possibility that in the future someone discovers that induced entropion is not an exclusive sign of intermittent entropion. For the test to be considered positive, the induced entropion must remain stable with eye movements and blinking. We arbitrarily defined that an entropion should last for at least 3 minutes to be considered stable. This rule seems to be effective but needs scientific scrutiny. In normal eyelids, the test is not capable of inducing entropion. Yet, in relatively loose eyelids, it generates a false entropion that vanishes readily with a blink or an eye excursion. Further studies on the sensitivity and specificity of this test are still necessary to refine its diagnostic value. As far as we know, this test has not been published previously.

\section{Disclosure}

The authors report no conflicts of interest in this work.

\section{References}

1. Levine MR. Involutional entropion. Ophthalmic Pract. 1987;5(3): $118-139$. 
2. Schaefer AJ. Involutional entropion. In: Smith BC, Nesi FA, Levine MR, Lisman RD, editors. Ophthalmic Plastic and Reconstructive Surgery. St Louis: CV Mosby Co; 1983;1:546-555.

3. Dryden RM, Leibsohn J, Wobig JI. Senile entropion: a review with evaluation of a procedure. Arch Ophthalmol. 1978;96:1058-1064.
4. Kronish JW. Entropion. In: Chen WP, editor. Oculoplastic Surgery: The Essentials. New York: Thieme; 2001:41-43.

5. Yassur I, Karesh JW, Hirschbein MJ. Entropio, trichiasis and districhiasis. In: Agarwal S, editor. Step by Step Oculoplastic Surgery. New Delhi: Jaypee Brothers Medical Publishers; 2005:144-146.

\section{Publish your work in this journal}

Clinical Ophthalmology is an international, peer-reviewed journal covering all subspecialties within ophthalmology. Key topics include: Optometry; Visual science; Pharmacology and drug therapy in eye diseases; Basic Sciences; Primary and Secondary eye care; Patient Safety and Quality of Care Improvements. This journal is indexed on

\section{Dovepress}

PubMed Central and CAS, and is the official journal of The Society of Clinical Ophthalmology (SCO). The manuscript management system is completely online and includes a very quick and fair peer-review system, which is all easy to use. Visit http://www.dovepress.com/ testimonials.php to read real quotes from published authors. 